\title{
Asymmetrical Neural Substrates of Tactile Discrimination in Humans: A Functional Magnetic Resonance Imaging Study
}

\author{
Tokiko Harada, ${ }^{1,2}$ Daisuke N. Saito, ${ }^{2}$ Ken-Ichi Kashikura, ${ }^{3}$ Tetsuhiro Sato, ${ }^{3}$ Yoshiharu Yonekura, ${ }^{3}$ Manabu Honda, ${ }^{1}$ and \\ Norihiro Sadato ${ }^{1,2,4}$ \\ ${ }^{1}$ Department of Physiological Sciences, The Graduate University for Advanced Studies (Sokendai), Kanagawa 240-0193, Japan, ${ }^{2}$ National Institute for \\ Physiological Sciences, Okazaki, Aichi 444-8585, Japan, ${ }^{3}$ Biomedical Imaging Research Center, Fukui Medical University, Fukui 910-1193, Japan, and \\ ${ }^{4}$ Japan Science and Technology Corporation/Research Institute of Science and Technology for Society, Kawaguchi 332-0012, Japan
}

The left-hand advantage seen during tactile discrimination tasks suggests hemispheric-processing asymmetry, although its neural substrates are not well known. We used functional magnetic resonance imaging to evaluate the laterality of the neural substrates involved in tactile discrimination in 19 normal volunteers. Passive tactile discrimination tasks, along with appropriate control tasks, were performed with both the right and left hands to evaluate the effects of the hand used and hemispheric effects (i.e., laterality of the activation pattern). Regardless of the hand used, the right dorsolateral prefrontal cortex, posterior parietal cortex, pre-supplementary motor area, and rostral portion of the dorsal premotor cortex (PMdr) were activated asymmetrically during tactile discrimination. This confirms the previous finding of a right-sided asymmetry for tactile shape discrimination. Hand effects were found in the left caudal portion of PMd (PMdc) adjacent to the central sulcus, which showed prominent activation during right-handed but not left-handed discrimination tasks. This asymmetric activation in the left PMdc might be related to the asymmetric interhemispheric interaction during right-handed tactile discrimination.

Key words: dorsal premotor cortex (PMd); fMRI; interhemispheric transfer; spatial-attention network; tactile shape discrimination; blood flow

\section{Introduction}

It has been suggested that tactile pattern discrimination is a right hemisphere function (Carmon and Benton, 1969; Fontenot and Benton, 1971; Zaidel and Sperry, 1973). Fontenot and Benton (1971) found that patients with left hemisphere lesions were deficient in the perception of the direction of tactile stimulation of the right hand, whereas patients with right hemisphere lesions showed a bilateral impairment. This idea is consistent with previous studies of patients with complete cerebral commissurotomy, which showed a left-hand advantage for nonverbal tactile tasks (Milner and Taylor, 1972; Kumar, 1977; Dodds, 1978). Using a cross-modal geometric matching task, Dodds (1978) found that the right hemisphere is superior in terms of both speed and accuracy. Using a tactile modification of the Memory for Designs test, Kumar (1977) found that processing in the right hemisphere was markedly superior to the left hemisphere, indicating independent right hemisphere-based memories of spatial configurations. Because this right hemisphere superiority is not evident in subjects with an intact or remnant corpus callosum, interhemispheric transfer probably equalizes the performance by either

Received April 14, 2004; revised July 14, 2004; accepted July 20, 2004.

This work was supported in part by Grant-in Aid for Scientific Research B\#14380380 to N.S. from the Japan Society for the Promotion of Science and by Special Coordination Funds for Promoting Science and Technology from the Ministry of Education, Culture, Sports, Science and Technology, the Japanese Government.

Correspondence should be addressed to Dr. Norihiro Sadato, Section of Cerebral Integration, Department of Cerebral Research, National Institute for Physiological Sciences, Myodaiji, Okazaki, Aichi 444-8585, Japan. E-mail: sadato@nips.ac.jp.

DOI:10.1523/JNEUROSCI.1395-04.2004

Copyright $\odot 2004$ Society for Neuroscience $\quad$ 0270-6474/04/247524-07\$15.00/0 hand. This implies that the neural substrates of tactile spatial processing might be right lateralized even if the left-hand advantage is not evident; furthermore, the right-hand process might be more demanding than the left-hand process because of interhemispheric transfer. However, the neural substrates of this compensatory workload are unknown.

Recent advances in neuroimaging techniques allow the visualization of task-related neural circuits using blood flow changes as an indirect index of neural activity. In the present study, we hypothesized that the difference between the neural substrates for tactile discrimination by the right and left hands might represent those regions necessary for interhemispheric transfer. To test this hypothesis, we conducted a functional magnetic resonance imaging (fMRI) study. A Braille letter discrimination task was performed by sighted subjects with no knowledge of Braille reading and hence was regarded as a non-lexical tactile discrimination task. To exclude any exploratory movements during tactile discrimination, the tactile stimuli were presented passively. To visualize the neural substrates of tactile discrimination, the discrimination task was contrasted with a nondiscrimination task; this allowed us to control for somatosensory input and motor responses. These tasks were performed using both the right and left hands. The hemisphere effect (i.e., laterality of the activation pattern), hand effect, and the interaction between these two were evaluated.

\section{Subjects and Methods}

Subjects

We studied a total of 19 healthy right-handed subjects, 8 females and 11 males, with a mean age of $24.8 \pm 3.6$ years. The subjects were all right- 
handed according to the Edinburgh handedness inventory (Oldfield, 1971). There was no history of neurological or psychiatric illness in any of the subjects, and none had any neurological deficits. All participants were naive to Braille reading. The protocol was approved by the ethical committee of Fukui Medical University and the National Institute for Physiological Sciences, Japan, and all subjects gave their written informed consent. Eight of the participants had taken part in previous studies that used identical tasks (Sadato et al., 2002).

\section{Magnetic resonance imaging}

A time course series of 126 volumes was acquired using T2*-weighted, gradient echo, echo planar imaging (EPI) sequences with a 3.0 Tesla MR imager (Signa Horizon; General Electric, Milwaukee, WI). Each volume consisted of 36 slices, with a slice thickness of $3.5 \mathrm{~mm}$ and a $0.5 \mathrm{~mm}$ gap, which included the entire cerebral and cerebellar cortices. The time interval between two successive acquisitions of the same image was 3000 $\mathrm{msec}$, and the echo time was $30 \mathrm{msec}$. The flip angle was $90^{\circ}$. The field of view was $22 \mathrm{~cm}$. The in-plane matrix size was $64 \times 64$ pixels with a pixel dimension of $3.44 \times 3.44 \mathrm{~mm}$. Tight, but comfortable, foam padding was placed around each subject's head to minimize head movement.

For anatomical reference, T2-weighted fast-spin echo images were obtained from each subject with location variables identical to those of the EPIs. In addition, high-resolution whole-brain MRIs were obtained with a conventional T2-weighted fast-spin echo sequence. A total of 112 transaxial images were obtained. The in-plane matrix size was $256 \times 256$ pixels, the slice thickness was $1.5 \mathrm{~mm}$, and the pixel size was $0.859 \times$ $0.859 \mathrm{~mm}$.

Tactile tasks

We used the same passive Braille tactile tasks as those used by Sadato et al. (2002) (see Fig. 1).

\section{Right hand}

Passive Braille tactile discrimination task. A session consisted of six task and six rest periods, each $30 \mathrm{sec}$ in duration, and alternating the task and rest periods. Braille stimuli were presented passively using a plastic rail on which different pairs of two-dot standard Braille characters (center-tocenter distance, $5 \mathrm{~mm}$ ) were printed. The rail was $1.7 \mathrm{~m}$ long. The rail was moved manually by an examiner from outside of the MRI gantry by a skid ( $1 \mathrm{~m}$ in length), which was fixed on the left side of the subject's body. The subject placed the right arm across the chest, rested the thumb and four fingers at a fixed position on the skid, and placed the right index finger so that the finger pad rested on the rail (Sadato et al., 2002). The initial position of the rail was set so that the subject's right index finger was located between two consecutive pairs of Braille characters. The subject's left hand was placed on a button box connected to a microcomputer for recording the subject's response.

A pacemaking cue was projected onto a semitransparent screen hung $\sim 1.5 \mathrm{~m}$ from the subject's eyes. For this, a liquid crystal display projector (ELP-7200L; Epson, Tokyo, Japan) was connected to a personal computer (Dynabook with Windows 95; Toshiba, Tokyo, Japan), on which in-house software generated a visual cue (a small filled circle). To maintain eye position, the subject was requested to fixate on the cue circle throughout the session. For $18 \mathrm{sec}$ before a session, a yellow cue was presented to allow the subject time to position both hands. Then, during the tactile discrimination task, red and green cues, each $3 \mathrm{sec}$ in duration, were given alternately for $30 \mathrm{sec}$. When the red cue was on, the examiner slowly moved the rail to present passively a pair of two-dot Braille characters to the subject's finger pad. The rail was moved three times in $3 \mathrm{sec}$ : $30 \mathrm{~mm}$ in the head-to-foot direction for $1 \mathrm{sec}, 30 \mathrm{~mm}$ in the foot-to-head direction in the next second, and $30 \mathrm{~mm}$ again in the head-to-foot direction in the final second. The speed of presentation was $\sim 30 \mathrm{~mm} / \mathrm{sec}$. The rail moved quietly without making any task-related sound. The examiner also confirmed that the subject did not move the right index finger for exploration. When the green cue was on, the rail stopped moving, and the subject responded by pushing a button with the left index finger if the pair of characters was the same, or with the middle finger if the characters were different. Reaction times were not measured. A $30 \mathrm{sec}$ rest condition followed, in which red and green cues were given alternately, as in the task condition. When the red cue was on, no tactile stimulus was presented.

\section{Table 1. Predefined contrasts}

\begin{tabular}{|c|c|c|c|c|}
\hline \multirow{3}{*}{$\begin{array}{l}\text { Name of con- } \\
\text { trasts }\end{array}$} & \multicolumn{4}{|c|}{ Conditions } \\
\hline & \multicolumn{2}{|c|}{ Left hand } & \multicolumn{2}{|c|}{ Right hand } \\
\hline & Disc & Non-disc & Disc & Non-disc \\
\hline LD & 1 & 0 & 0 & 0 \\
\hline LN & 0 & 1 & 0 & 0 \\
\hline RD & 0 & 0 & 1 & 0 \\
\hline RN & 0 & 0 & 0 & 1 \\
\hline$L(D-N)$ & 1 & -1 & 0 & 0 \\
\hline$R(D-N)$ & 0 & 0 & 1 & -1 \\
\hline$(R-L)(D-N)$ & -1 & 1 & 1 & -1 \\
\hline$(R+L)(D-N)$ & 1 & -1 & 1 & -1 \\
\hline
\end{tabular}

Disc, Discrimination; Non-disc, nondiscrimination.

When the green cue was on, the subject pushed buttons with the left index and middle finger alternately. The comparison of neuroimages collected during the discrimination task versus those during rest periods thus allowed the correction for the effects of the cue and response movements.

Passive Braille tactile nondiscrimination task. In the tactile nondiscrimination task, which was used to control for sensorimotor effects, six-dot (instead of two-dot) Braille characters were presented when the red cue was given. When the green cue was on, the subject pushed buttons with the left index and middle finger alternately. The other variables were identical to those in the Braille tactile discrimination task.

\section{Left hand}

The aforementioned tasks also were performed with the left hand. The order of the conditions was counterbalanced within the group. Before scanning, outside of the MRI room, the subjects sufficiently practiced the tactile discrimination task using different sets of two-dot Braille characters than those used in each task.

\section{Data analysis}

The first six volumes of each fMRI session were discarded because of unsteady magnetization, and the remaining 120 volumes per session ( 480 volumes per subject) were used for analysis. The data were analyzed using statistical parametric mapping (SPM99; Wellcome Department of Cognitive Neurology, London, UK) implemented in Matlab (Mathworks, Sherborn, MA) (Friston et al., 1994, 1995a,b). After realignment, all images were coregistered to the high-resolution three-dimensional T2weighted MRI with use of the anatomical MRI with T2-weighted spinecho sequences from identical locations to the fMRI images. The parameters for affine and nonlinear transformation into a template of T2weighted images that was already fit for a standard stereotaxic space (Montreal Neurological Institute template) (Evans et al., 1994) were estimated based on the high-resolution three-dimensional T2-weighted MRI using least-squares means (Friston et al., 1995a). The parameters were applied to the coregistered fMRI data. The anatomically normalized fMRI data were filtered using a Gaussian kernel of $10 \mathrm{~mm}$ (full width at half-maximum) in the $x, y$, and $z$ axes.

\section{Statistical analysis}

Statistical analysis was conducted at two levels. First, individual taskrelated activation was evaluated. Second, to make inferences at a population level, individual data were summarized and incorporated into a random effect model (Friston et al., 1999).

\section{Individual analysis}

The signal was scaled proportionally by setting the whole-brain mean value to 100 arbitrary units. The signal time course of each subject, with 480 time points, was modeled with four boxcar functions convolved with a hemodynamic response function, high-pass filtering $(120 \mathrm{sec})$, and session effects. To test hypotheses about regionally specific condition effects, the estimates for each condition were compared by means of the linear contrasts shown in Table 1 . The resulting set of voxel values for each comparison constituted a statistical parametric map (SPM) of the $t$ statistic $[\operatorname{SPM}\{t\}]$. The threshold for the $\operatorname{SPM}\{t\}$ was set at a false- 
discovery rate (FDR) of $p<0.01$ (Genovese et al., 2002). FDR is the proportion of false positives (incorrect rejections of the null hypothesis) among multiple voxel-wise tests for which the null hypothesis is rejected, and hence the procedure controls the family-wise error rate (Genovese et al., 2002). The activation foci depicted by this height threshold were then tested by their spatial extent, based on the theory of Gaussian random field considering clusters as "rare events" that occur in a whole brain according to the Poisson distribution (Friston et al., 1996). Statistical threshold for the spatial extent test was set at $p<0.05$ (Friston et al., 1996).

When evaluating the neural substrates of the tactile discrimination processes, we controlled for nonspecific somatosensory processes (D-N), hand effect [right (R) versus left (L)], and hemispheric effects. To evaluate the effects of hand use, comparisons of $(R-L)(D-N)$ (Table 1) were performed within the areas activated during the right-handed (D-N) condition ( $p<0.05$; spatial extent test). The threshold for the $\operatorname{SPM}\{t\}$ was set at an FDR of $p<0.01$ for clusters larger than 40 voxels (Genovese et al., 2002). We omitted the spatial extent test (Friston et al., 1996) because the spatial extent test is valid only for large search regions (Worsley et al., 1996).

\section{Group analysis with random-effect model}

The weighted sum of the parameter estimates in the individual analysis constituted "contrast" images, which were used for the group analysis (Friston et al., 1999). The contrast images obtained by individual analyses represent the normalized task-related increment of the MR signal of each subject [i.e., the discrimination task vs rest period (D), the nondiscrimination task vs rest period $(\mathrm{N})$, and the discrimination vs nondiscrimination tasks (D-N)]. A total of 19 subjects with three contrasts (discrimination, nondiscrimination, and discrimination-nondiscrimination) each for the right- and left-hand conditions were used for analysis. The resulting set of voxel values for each contrast constituted an SPM $\{t\}$. The SPM $\{t\}$ was transformed to normal distribution units $[\operatorname{SPM}\{Z\}]$. The threshold for the $\operatorname{SPM}\{t\}$ was set at an FDR of $p<0.01$ (Genovese et al., 2002). Statistical threshold for the spatial extent test on the clusters was set at $p<0.05$ (Friston et al., 1996), as in the individual analysis.

To evaluate the effects of hand use, contrast images of the (D-N) (one image per subject) for the right-hand condition $[R(D-N)]$ (Table 1) were compared with those for the left-hand condition $[L(D-N)]$ (Table 1). We did this in a pairwise manner within the areas activated during the right-handed ( $D-N)$ condition ( $p<0.05$; spatial extent test). To evaluate the hemisphere effects on tactile discrimination, contrast images of $(L+$ $R)(D-N)$ (Table 1) were flipped in the horizontal (right-left) direction. Asymmetric involvement of the neural substrates for the discrimination task, regardless of the hand used, was shown by the comparison between unflipped and flipped groups in a pair-wise manner. The test was performed within the regions that showed activation during this task when either hand was used. The threshold for the SPM $\{t\}$ was set at an FDR of $p<0.01$ for clusters larger than 40 voxels (Genovese et al., 2002).

\section{Results}

\section{Task performance}

The passive tactile discrimination tasks were performed equally well by the subjects when using either the right hand or the left hand. Right-handed accuracy was $67.4 \pm 7.6 \%$, and left-handed accuracy was $64.0 \pm 12.5 \%$, with no significant difference between these scores $(n=19 ; p=0.24$; paired $t$ test $)$.

\section{Group analysis with random-effect model}

\section{Nondiscrimination}

The passive nondiscrimination tasks activated the contralateral primary sensory motor area (SM1) and the dorsal portion of the lateral premotor area, the bilateral secondary somatosensory area (SII) extending to the postcentral gyrus (GPoC), the anterior intraparietal sulcus (IPA), the ipsilateral superior parietal lobule (LPs), and the cerebellum, regardless of the hand used (Fig. 1; also see supplemental material, available at www.jneurosci.org/cgi/



Figure 1. Task design. During the discrimination task, pairs of two-dot standard Braille characters were presented passively to the index finger of one hand when the red cue (shown as black) was on. When the green cue (shown as gray) was on, the subject responded by pushing a button with the other index finger (I) if the pairwise characters were the same, or with the middle finger $(M)$ if the characters were different. During the rest condition, no tactile stimulus was presented. When the green cue was on, the subject pushed buttons with the left index and middle finger alternately. During the nondiscrimination session, pairs of six-dot standard Braille characters were presented, and no discrimination was requested.

content/full/24/34/7524/DC1). The supplementary motor area (SMA) was active with right-handed passive tactile stimulation, but this was not true when the left hand was used. The activation in the dorsal portion of the premotor cortex was designated as dorsal premotor cortex (PMd) according to the criteria of Rizzolatti et al. (2002) (Talairach's $z$-coordinate, $>50 \mathrm{~mm}$ ).

\section{Discrimination}

The passive discrimination task activated bilateral SM1 and PMd extending to the SMA, SII extending to the GPoC, IPA extending to the posterior intraparietal sulcus (IPP), the frontal cortices, and the ipsilateral cerebellum, regardless of the hand used (Fig. 1; see also supplemental material, available at www.jneurosci.org/ cgi/content/full/24/34/7524/DC1). The contralateral cerebellum was activated during right-handed processing.

\section{Discrimination-nondiscrimination $(D-N)$}

When the discrimination condition was contrasted with the nondiscrimination (D-N) task, with the exception of SM1 and SII, the following areas that were activated by the nondiscrimination task showed increased activation regardless of the hand used: the bilateral IPA, cerebellum, and SMA (Figs. 2, 3). With the right hand, the PMd was activated bilaterally, whereas with the left hand, its task-related activity was right lateralized. In addition to the tactile-related areas defined by the nondiscrimination task, discrimination tasks activated bilateral prefrontal cortices regardless of the hand used, and the IPP was activated bilaterally with the right hand, whereas the right IPP was active when the left hand was used (supplemental material, available at www.jneurosci.org/cgi/ content/full/24/34/7524/DC1).

\section{Hemisphere effect}

Right-lateralized activities were found in the IPP, rostral PMd, dorsolateral prefrontal cortex, anterior insula, and pre-SMA (Fig. 4). These areas showed consistent right-lateralized activation during performance of the task with both the right and left hands. Left-lateralized activity was found in the rostral PMd. 
Discrimination

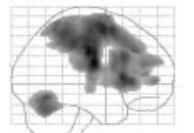

$\mathrm{LH}$
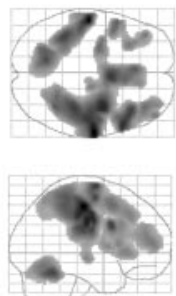

$\mathrm{RH}$
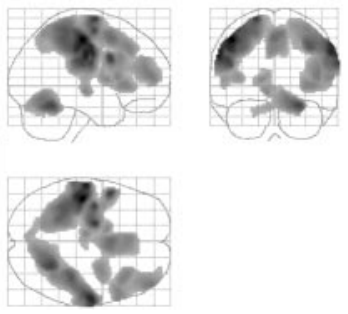

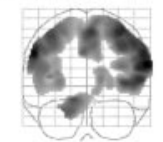

Non-discrimination
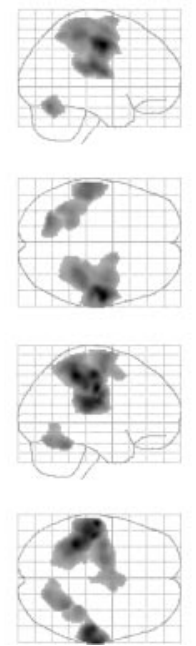
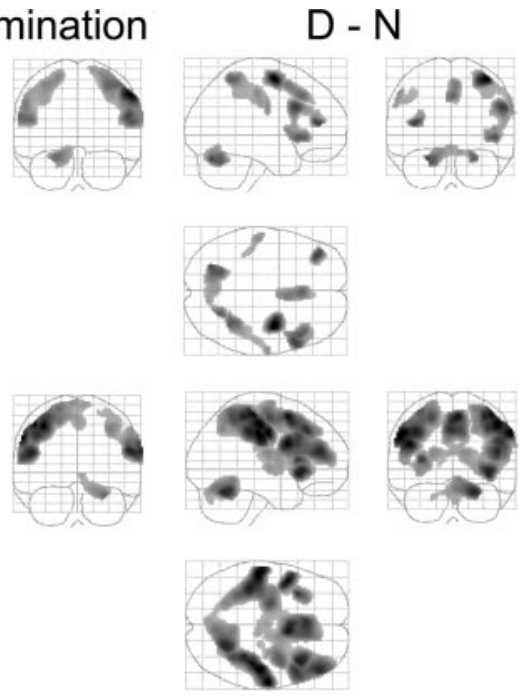

Discussion

Nondiscrimination condition

Activation of the SM1, SII, PMd, SMA, and cerebellum by passive tactile stimulation suggests that the cortical motor networks participate in somatosensory processing (Romo and Salinas, 2001). In the task epoch, the subjects were asked to respond to tactile stimuli by making a button press. In the rest epoch, the subjects pushed the button without tactile stimuli. Hence, the former contains a sensorimotor link that might account for the PMd and SMA activity.

\section{D-N condition}

IPA activation with absent SII activation during shape discrimination

This study showed that the passive nondiscriminatory tactile task activated SII, with no additional activation during the discriminatory task. However, the IPA bilaterally showed more activation during passive tactile discrimination compared with the nondiscrimination condition (Fig. 3). This is consistent with the findings of Ro-
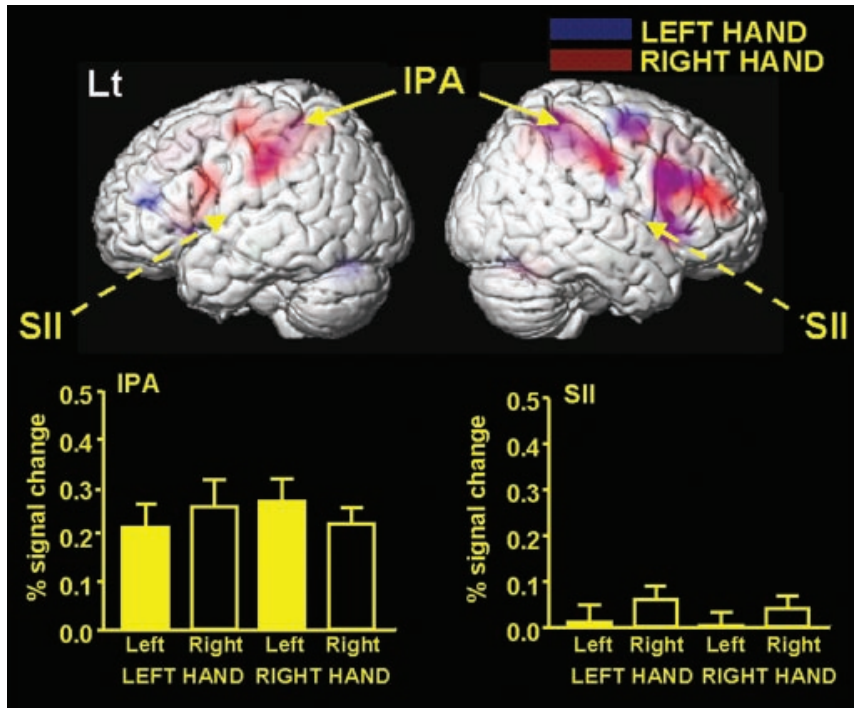

Figure 3. Statistical parametric map of the average neural activity within the group during the discrimination task compared with the activity during the nondiscrimination task (D-N). The activities while performing the task with the left (blue) and right (red) hands were superimposed on surface-rendered high-resolution MRIs unrelated to the subjects of the present study, viewed from the left and right. Bottom left, The averaged percentage of signal change of D-N in the bilateral IPA $( \pm 36,-40,50)$. Bottom right, The averaged percentage of signal changes in the bilateral $\mathrm{SII}(-50,-24,20)$ and $(56,-16,20)$. Percentage of signal change was calculated individually within spherical volumes of interest with a diameter of $10 \mathrm{~mm}$ placed at the center of the volume. These data were presented as the mean \pm SEM of 19 subjects. Lt, Left.

\section{Hand effect}

Direct comparison between the task-related activation depicted by the $\mathrm{D}-\mathrm{N}$ contrast with the right hand with that of the left hand showed more prominent activation in the left precentral gyrus, corresponding to the caudal PMd (Fig. 5). Reverse contrast did not show any significant activation. This hand effect was observed consistently in individual analyses. Three representative subjects are shown in Figure 6. There was no significant activation for the reverse contrast [i.e., $L(D-N)-R(D-N)$ ]. land et al. (1998) that roughness discrimination activated SII significantly more than length or shape discrimination. Conversely, shape and length discrimination activated the same cortical area lining the IPA more than roughness discrimination. They suggested that there are different cortical processing streams for somatosensory submodalities, such as microgeometry (texture) and macrogeometry (shape and length).

\section{Cerebellum}

The anterior lobe of the cerebellum showed more activation during the discrimination task than during the nondiscrimination task. Because this was observed during passive conditions, the cerebellar activation might be related to the nonmotor cognitive process of shape discrimination. Cognitive tasks are known to activate cerebellar structures (Petersen et al., 1988; Kim et al., 1994; Raichle et al., 1994; Fiez et al., 1996; Gao et al., 1996; Cabeza and Nyberg, 2000; Liu et al., 2000).

\section{Hemisphere effect}

This study revealed right-lateralized activation of the parietal, prefrontal, and dorsal premotor cortices regardless of the hand performing the task.

\section{Posterior intraparietal sulcus} discrimination. The posterior parietal cortex [Brodmann area (BA) 7/40], particularly the intraparietal sulcus, consists of multiple subdivisions, each of which is involved in particular aspects of visual or somatosensory information processing. The posterior parietal cortex and BA 6 are connected in a specific pattern, forming several frontoparietal circuits (Rizzolatti et al., 1998; Geyer et al., 2000). These two cortical areas function in concert during cognitive operations, motor control (Deiber et al., 1997), and voluntary attentional control (Hopfinger et al., 2000).

\section{Dorsal premotor cortex}

The PMd is the dorsolateral subdivision of BA 6, which is defined as the agranular frontal cortex situated between the primary mo-
We found right-lateralized activation in the IPP during tactile 
tor cortex (M1) and the prefrontal cortex. In stereotactic space, the boundary of the PMd and ventral premotor cortex is said to be at $Z=+50$ (Rizzolatti et al., 2002). At the level of the hand, M1 is located near $Z=50$; hence, the convolution of the precentral gyrus corresponds mostly to the caudal PMd (PMdc), because the representation of the hand in M1 is located in the central sulcus (Yousry et al., 1997). The rostral PMd (PMdr) is probably located anterior to the superior precentral sulcus (Rizzolatti et al., 1998). In addition, the vertical anterior-commissural plane was used as a landmark of the border between the PMdc and PMdr (Deiber et al., 1991). There might be functional segregation within the PMd in a rostrocaudal direction in primates (Geyer et al., 2000). The PMdc is more closely related to motor execution, whereas the PMdr is involved more with the sensory components of motor tasks (Weinrich and Wise, 1982; Johnson et al., 1996; Shen and Alexander, 1997; Hanakawa et al., 2002). The PMd receives input from the somatosensory areas in the parietal cortex. Non-human primate studies showed that the PMdc receives input from area 5 in the dorsal bank of the intraparietal sulcus (Chavis and Pandya, 1976) and the caudal part of area 7. In contrast, the PMdr receives inputs from areas $7 \mathrm{~m}$, 7ip, and the superior temporal sulcus (Kurata, 1991). Furthermore, the PMdr receives projections from the prefrontal cortex, which receives projections from the inferior parietal area 7a (Barbas and Pandya, 1987; Tanne et al., 1995), forming the parietal-prefrontal-premotor networks. These inputs presumably represent the sensoryaspects of the set-related activity observed in the PMd and are more dominant in the PMdr than PMdc (Tanne et al., 1995). Furthermore, recent functional neuroimaging studies indicate that the PMd might have nonmotor cognitive functions (Jonides et al., 1993; Deiber et al., 1998; Hanakawa et al., 2002, 2003).

\section{Right lateralization}

A previous positron emission tomographic study (Sadato et al., 1998) revealed that blind subjects showed activation of the right dorsal premotor cortex and the right prefrontal cortex during tactile discrimination tasks, regardless of the finger used for reading. O'Sullivan et al. (1994) suggested that the right PMd is involved in length discrimination, probably through the close interplay between sensory and motor regions during active touch. Gitelman et al. (1996) have shown that exploratory tasks with the right hand activate the right cingulate, dorsal premotor, and posterior parietal areas; they attributed this to the spatial-attention requirements of the task. Because the right dorsal premotor, posterior parietal, and prefrontal cortices are related to visuospatial

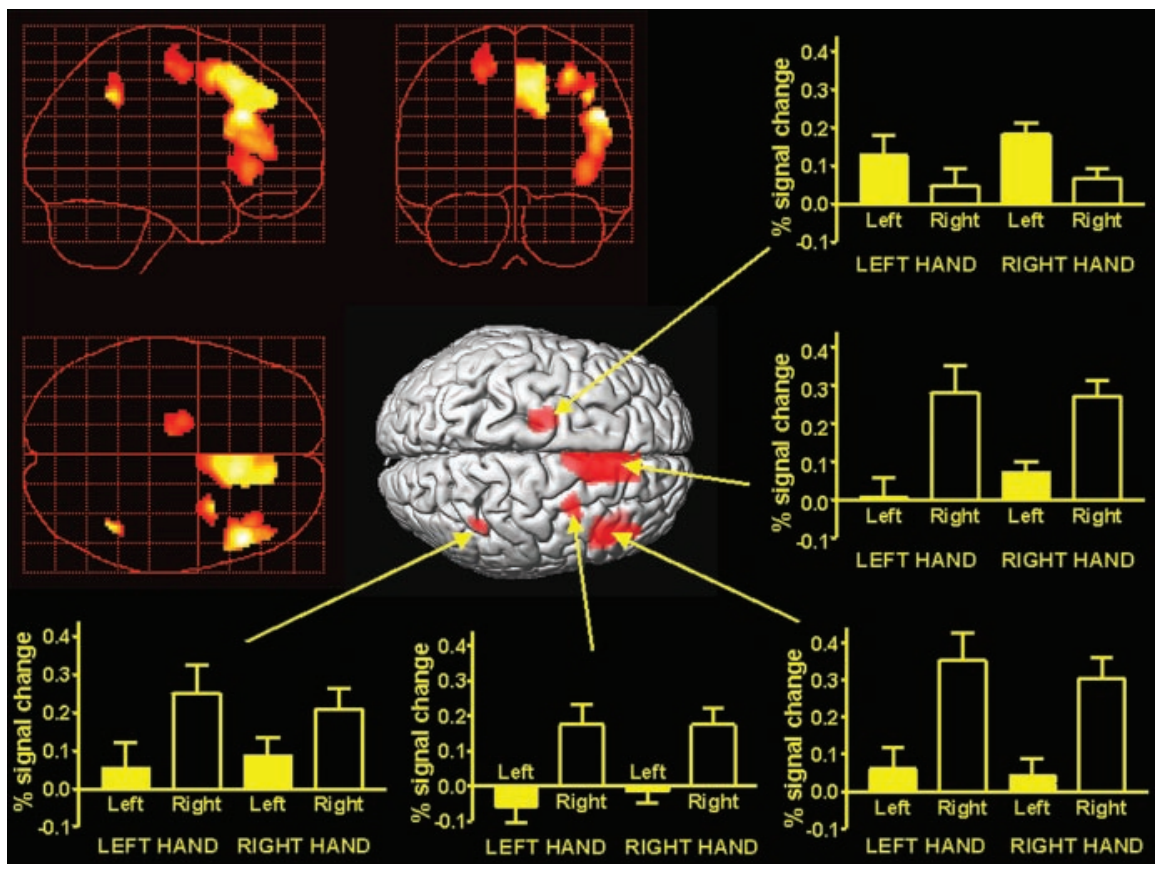

Figure 4. Asymmetric neural representation of tactile discrimination by either hand. The contrast images of (D-N) were compared with those flipped in the horizontal (right-left) direction in a pairwise manner (see Table 1). The test was performed within the areas that revealed activation by the (D-N) condition with either hand. The SPM was superimposed on a surface(center). The three-dimensional information was collapsed into two-dimensional sagittal, coronal, and transverse images viewed from the right (top left), back (top middle), and top of the brain (middle row, left). The percentage of signal changes in dorsolateral prefrontal cortex $( \pm 50,26,30)$ (bottom right), pre-SMA $( \pm 10,22,48)$ (middle row, right), IPP $( \pm 42,-48,42)$ (bottom left), and PMdr $( \pm 32,8,52)$ (bottom middle) and $( \pm 20,-10,56)$ (top right) were presented as the mean \pm SEM of 19 subjects.

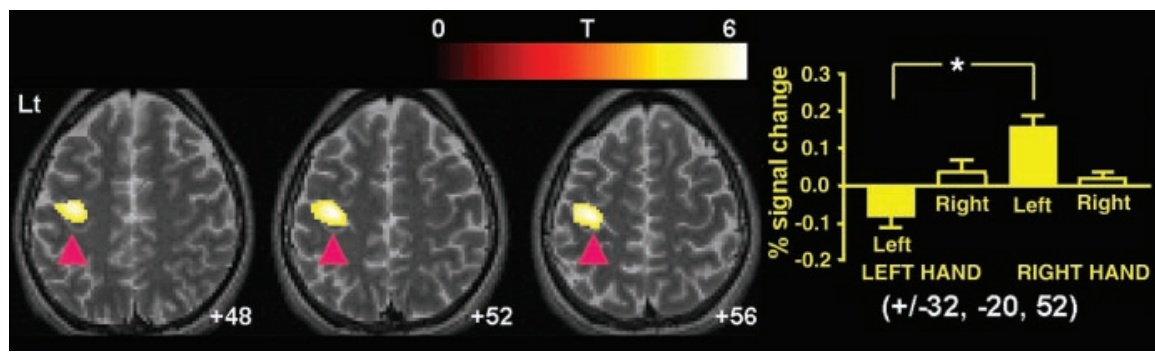

Figure 5. The SPM of the average neural activity in the $(D-N)$ condition with the right hand compared with the left hand, within the activated areas in the (D-N) condition with the right hand. The focus of activation was superimposed on the transaxial plane the inverted-omega shape that is a landmark of the hand area. The averaged percentage of signal change in the precentra activated the left precentral gyrus more prominently than discrimination by the left hand $\left({ }^{*} t=5.98\right.$; paired Student's $t$ test; $\mathrm{df}=$ 18). Its right counterpart was not active during tactile discrimination by either hand. There was significant hand by hemisphere interaction in this area (paired Student's $t$ test; $t=5.4$; df $=18$ ). Lt, Left.

working memory (Jonides et al., 1993), they are components of a functional network for modality-independent extrapersonal spatial attention, which might be required for exploratory finger movements. This study, however, revealed that without active exploratory movement, tactile discrimination activated the rightdominant parietal-premotor-prefrontal networks, regardless of the hand used to perform the task. This is consistent with a previous study showing that the right parietal-premotor-prefrontal network was activated by passive tactile discrimination performed with the right hand (Bodegard et al., 2001). Gitelman et al. (1999) showed that the right premotor and posterior parietal areas are specialized for spatial attention with stringent controls 
(R-L)(D-N)

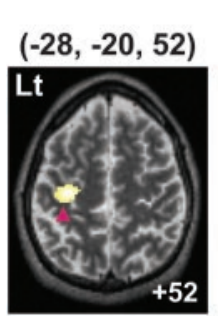

\section{Left PMdc}


$(-34,-14,56)$
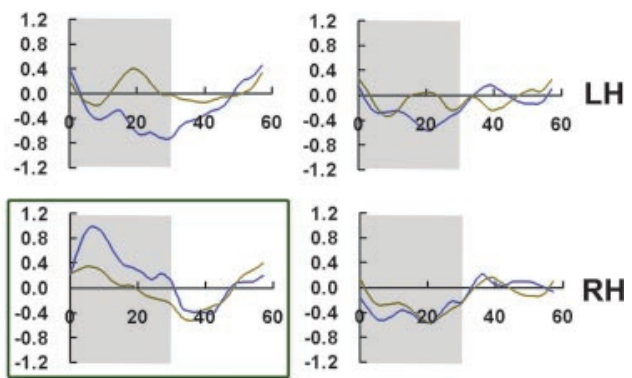

Figure 6. Individual analysis of the hand effect on the passive tactile discrimination. The foci with more prominent activation during tactile discrimination with the right hand than with the left hand (left column), indicated by means of the comparison of $(R-L)(D-N)$, were superimposed on the high-resolution T2-weighted MRI of each individual. The arrowheads indicate the central sulcus. The activated foci are on the left precentral gyrus. The Talairach's coordinates of the area with maximum intensity change are shown. The task-related activation of the left PMdc (middle column) and the right PMdc (right column) during the task phase (shaded; $30 \mathrm{sec}$ in duration) when compared with the following rest phase (nonshaded; $30 \mathrm{sec}$ ). The percentage of signal changes averaged across the six repeated task-rest periods of the discrimination session (blue line) and the nondiscrimination session (green line) were plotted. LH, Left hand; $\mathrm{RH}$, right hand; Lt, left.

for response-related motor activity, motor inhibition, eye movements, and working memory. Hence, the right-lateralized neural substrates for spatial attention might contribute to the right hemisphere dominance found during the tactile shape discrimination task.

\section{Hand effects}

This study revealed that the hand that performed the task influenced activity in the left PMdc only: this activation during use of the right hand was left lateralized, and there was no activation while the left hand was used (Fig. 5). During the nondiscrimination condition, the PMdc revealed the activation by the contralateral hand but not by the ipsilateral hand (Fig. 2), and hence the differential left-right hand effect occurs during the discrimination task. This activation pattern is unlikely to be related to movement control. First, the tactile stimuli were presented passively in both the discrimination and control conditions, eliminating any exploratory movements of the stimulated fingers. Second, the responses by the hand contralateral to the stimulated side during the tactile discrimination task were controlled for by the nondiscrimination condition in which the subjects were required to alternate finger movements. It should be noted that the tactile discrimination task required that the button press was based on the tactile stimuli. This is conditional motor behavior guided by sensory cues, which is not included in the control condition. Both the PMdr and PMdc are important in conditional motor behavior guided by symbolic cues (Petrides, 1986; Passingham, 1988; Wise and Murray, 2000). This might represent an interface between the output of the sensory categorization process and the motor command used to indicate the movement choice (Romo and Salinas, 2001). However, asymmetric left PMdc activation cannot be explained by the conditional motor behavior as guided by tactile cues, because the button press was performed by the left hand during right-hand discrimination. If the PMdc activation were attributable to conditional motor behavior, it should have appeared in the PMdc ipsilateral to the stimulated hand. Hence, asymmetric left PMdc activation might represent nonmotor processing. Considering these anatomical and functional connections, the activation of the left PMdc only by right-handed discrimination may represent the output of the sensory categorization process; this might be part of the parieto-premotor networks in the left hemisphere that are driven by tactile information from the right hand.

Several lines of evidence suggest that the PMd is involved in the interhemispheric interaction. The PMd has dense corticocortical connections with the SMA (Kurata, 1991), which in turn has dense and widespread transcortical connections with the contralateral SMA and the premotor cortex (Rouiller et al., 1994). The PMd is related to the interhemispheric interaction seen during the performance of bimanual coordinated movements (Sadato et al., 1997; Kermadi et al., 2000; Immisch et al., 2001; Gerloff and Andres, 2002). Certainly, this does not mean that the PMdc is the only area involved in the interhemispheric interaction during tactile tasks. Tactile information is represented bilaterally in the postcentral gyrus and its posterior extension (Iwamura, 1998). Partial callosotomy sparing the splenium of the corpus callosum did not induce right hemisphere superiority, whereas complete callosotomy did (Kumar, 1977), suggesting that interhemispheric interaction occurs at the level of the parietal cortex. Hence, the increased activity of the left PMdc during right-hand discrimination might represent the additional workload necessary for the parieto-premotor network on the left to access the right-lateralized neural resources for spatial attention.

\section{References}

Barbas H, Pandya DN (1987) Architecture and frontal cortical connections of the premotor cortex (area 6) in the rhesus monkey. J Comp Neurol 256:211-228.

Bodegard A, Geyer S, Grefkes C, Zilles K, Roland PE (2001) Hierarchical processing of tactile shape in the human brain. Neuron 31:317-328.

Cabeza R, Nyberg L (2000) Imaging cognition. II. An empirical review of 275 PET and fMRI studies. J Cogn Neurosci 12:1-47.

Carmon A, Benton AL (1969) Tactile perception of direction and number in patients with unilateral cerebral disease. Neurology 19:525-532.

Chavis DA, Pandya DN (1976) Further observations on corticofrontal connections in the rhesus monkey. Brain Res 117:369-386.

Deiber M-P, Passingham RE, Colebatch JG, Friston KJ, Nixon PD, Frackowiak RSJ (1991) Cortical areas and the selection of movement: a study with positron emission tomography. Exp Brain Res 84:393-402.

Deiber M-P, Wise SP, Honda M, Catalan MJ, Grafman J, Hallett M (1997) Frontal and parietal networks for conditional motor learning: a positron emission tomography study. J Neurophysiol 78:977-991. 
Deiber M-P, Ibanez V, Honda M, Sadato N, Raman R, Hallett M (1998) Cerebral processes related to visuomotor imagery and generation of simple finger movements studied with positron emission tomography. NeuroImage 7:73-85.

Dodds AG (1978) Hemispheric differences in tactuo-spatial processing. Neuropsychologia 16:247-254.

Evans AC, Kamber M, Collins DL, MacDonald D (1994) An MRI-based probabilistic atlas of neuroanatomy. In: Magnetic resonance scanning and epilepsy (Shorvon SD, ed), pp 263-274. New York: Plenum.

Fiez JA, Raife EA, Balota DA, Schwarz JP, Raichle ME, Petersen SE (1996) A positron emission tomography study of the short-term maintenance of verbal information. J Neurosci 16:808-822.

Fontenot DJ, Benton AL (1971) Tactile perception of direction in relation to hemispheric locus of lesion. Neuropsychologia 9:83-88.

Friston KJ, Jezzard P, Turner R (1994) Analysis of functional MRI timeseries. Hum Brain Mapp 1:153-171.

Friston KJ, Ashburner J, Frith CD, Heather JD, Frackowiak RSJ (1995a) Spatial registration and normalization of images. Hum Brain Mapp 2:165-188.

Friston KJ, Holmes AP, Worsley KJ, Poline J-B, Frith CD, Frackowiak RSJ (1995b) Statistical parametric maps in functional imaging: a general linear approach. Hum Brain Mapp 2:189-210.

Friston KJ, Holmes A, Poline J-B, Price CJ, Frith CD (1996) Detecting activations in PET and fMRI: levels of inference and power. NeuroImage 4:223-235.

Friston KJ, Holmes AP, Worsley KJ (1999) How many subjects constitute a study? NeuroImage 10:1-5.

Gao J-H, Parsons LM, Bower JM, Xiong J, Li J, Fox PT (1996) Cerebellum implicated in sensory acquisition and discrimination rather than motor control. Science 272:545-547.

Genovese CR, Lazar NA, Nichols T (2002) Thresholding of statistical maps in functional neuroimaging using the false discovery rate. NeuroImage 15:870-878.

Gerloff C, Andres FG (2002) Bimanual coordination and interhemispheric interaction. Acta Psychol (Amst) 110:161-186.

Geyer S, Matelli M, Luppino G, Zilles K (2000) Functional neuroanatomy of the primate isocortical motor system. Anat Embryol (Berl) 202:443-474.

Gitelman DR, Alpert NM, Kosslyn S, Daffner K, Scinto L, Thompson W, Mesulam M-M (1996) Functional imaging of human right hemispheric activation for exploratory movements. Ann Neurol 39:174-179.

Gitelman DR, Nobre AC, Parrish TB, LaBar KS, Kim YH, Meyer JR, Mesulam M (1999) A large-scale distributed network for covert spatial attention: further anatomical delineation based on stringent behavioural and cognitive controls. Brain 122:1093-1106.

Hanakawa T, Honda M, Sawamoto N, Okada T, Yonekura Y, Fukuyama H, Shibasaki H (2002) The role of rostral Brodmann area 6 in mentaloperation tasks: an integrative neuroimaging approach. Cereb Cortex 12:1157-1170.

Hanakawa T, Honda M, Okada T, Fukuyama H, Shibasaki H (2003) Differential activity in the premotor cortex subdivisions in humans during mental calculation and verbal rehearsal tasks: a functional magnetic resonance imaging study. Neurosci Lett 347:199-201.

Hopfinger JB, Buonocore MH, Mangun GR (2000) The neural mechanisms of top-down attentional control. Nat Neurosci 3:284-291.

Immisch I, Waldvogel D, van Gelderen P, Hallett M (2001) The role of the medial wall and its anatomical variations for bimanual antiphase and in-phase movements. NeuroImage 14:674-684.

Iwamura Y (1998) Hierarchical somatosensory processing. Curr Opin Neurobiol 8:522-528.

Johnson PB, Ferraina S, Bianchi L, Caminiti R (1996) Cortical networks for visual reaching: physiological and anatomical organization of frontal and parietal lobe arm regions. Cereb Cortex 6:102-119.

Jonides J, Smith EE, Koeppe RA, Awh E, Minoshima S, Mintun MA (1993) Spatial working memory in humans as revealed by PET. Nature 363:623-625.

Kermadi I, Liu Y, Rouiller EM (2000) Do bimanual motor actions involve the dorsal premotor (PMd), cingulate (CMA) and posterior parietal (PPC) cortices? Comparison with primary and supplementary motor cortical areas. Somatosens Mot Res 17:255-271.
Kim SG, Ugurbil K, Strick PL (1994) Activation of a cerebellar output nucleus during cognitive processing. Science 265:949-951.

Kumar S (1977) Short term memory for a nonverbal tactual task after cerebral commissurotomy. Cortex 13:55-61.

Kurata K (1991) Corticocortical inputs to the dorsal and ventral aspects of the premotor cortex of macaque monkeys. Neurosci Res 12:263-280.

Liu Y, Pu Y, Gao J-H, Parsons L, Xiong J, Liotti M, Bower JM, Fox PT (2000) The human red nucleus and lateral cerebellum in supporting roles for sensory information processing. Hum Brain Mapp 10:147-159.

Milner B, Taylor L (1972) Right-hemisphere superiority in tactile patternrecognition after cerebral commissurotomy: evidence for nonverbal memory. Neuropsychologia 10:1-15.

Oldfield RC (1971) The assessment and analysis of handedness: the Edinburgh inventory. Neuropsychologia 9:97-113.

O’Sullivan BT, Roland PE, Kawashima R (1994) A PET study of somatosensory discrimination in man: microgeometry versus macrogeometry. Eur J Neurosci 6:137-148.

Passingham RE (1988) Premotor cortex and preparation for movement. Exp Brain Res 70:590-596.

Petersen SE, Fox PT, Posner MI, Mintun M, Raichle ME (1988) Positron emission tomographic studies of the cortical anatomy of single-word processing. Nature 331:585-589.

Petrides M (1986) The effect of periarcuate lesions in the monkey on the performance of symmetrically and asymmetrically reinforced visual and auditory go, no-go tasks. J Neurosci 6:2054-2063.

Raichle ME, Fiez JA, Videen TO, MacLeod A-MK, Pardo JV, Fox PT, Petersen SE (1994) Practice-related changes in human brain functional anatomy during nonmotor learning. Cereb Cortex 4:8-26.

Rizzolatti G, Luppino G, Matelli M (1998) The organization of the cortical motor system: new concepts. Electroencephalogr Clin Neurophysiol 106:283-296.

Rizzolatti G, Fogassi L, Gallese V (2002) Motor and cognitive functions of the ventral premotor cortex. Curr Opin Neurobiol 12:149-154.

Roland PE, O'Sullivan B, Kawashima R (1998) Shape and roughness activate different somatosensory areas in the human brain. Proc Natl Acad Sci USA 95:3295-3300

Romo R, Salinas E (2001) Touch and go: decision-making mechanisms in somatosensation. Annu Rev Neurosci 24:107-137.

Rouiller EM, Balalian A, Kazennikov O, Moret V, Yu X-H, Wiesendanger M (1994) Transcallosal connections of the distal forelimb representation of the primary and supplementary motor cortical areas in macaque monkeys. Exp Brain Res 102:227-243.

Sadato N, Yonekura Y, Waki A, Yamada H, Ishii Y (1997) Role of the supplementary motor area and the right premotor cortex in coordination of bimanual movements. J Neurosci 17:9667-9674.

Sadato N, Pascual-Leone A, Grafman J, Deiber M-P, Ibanez V, Hallett M (1998) Neural networks for Braille reading by the blind. Brain 121:1213-1229.

Sadato N, Okada T, Honda M, Yonekura Y (2002) Critical period for crossmodal plasticity in blind humans: a functional MRI study. NeuroImage 16:389-400.

Shen L, Alexander GE (1997) Preferential representation of instructed target location versus limb trajectory in dorsal premotor area. J Neurophysiol 77:1195-1212.

Tanne J, Boussaoud D, Noelle B-Z, Rouiller EM (1995) Direct visual pathways for reaching movements in the macaque monkey. NeuroReport 7:267-272.

Weinrich M, Wise SP (1982) The premotor cortex of the monkey. J Neurosci 2:1329-1345.

Wise SP, Murray EA (2000) Arbitrary associations between antecedents and actions. Trends Neurosci 23:271-276.

Worsley KJ, Marrett S, Neelin P, Vandal AC, Friston KJ, Evans AC (1996) A unified statistical approach for determining significant signals in images of cerbral activation. Hum Brain Mapp 4:58-73.

Yousry T, Schmid U, Alkadhi H, Schmidt D, Peraud A, Buettner A, Winkler P (1997) Localization of the motor hand area to a knob on the precentral gyrus: a new landmark. Brain 120:141-157.

Zaidel D, Sperry RW (1973) Performance on the raven's colored progressive matrices test by subjects with cerebral commissurotomy. Cortex 9:34-39. 